\title{
Éloignement social pour lutter contre la COVID-19 : nous sommes tous en première ligne
}

\author{
Kirsten Patrick MB BCh MSc, Matthew B. Stanbrook MD PhD, Andreas Laupacis MD MSc
}

— Citation : CMAJ 2020 May 11;192:E516-7. doi : 10.1503/cmaj.200606-f; publication hâtive le 8 avril 2020

Voir la version anglaise de l'article ici : www.cmaj.ca/lookup/doi/10.1503/cmaj.200606; voir des articles connexes (en anglais) ici : www.cmaj.ca/lookup/doi/10.1503/cmaj.200457 et www.cmaj.ca/lookup/doi/10.1503/cmaj.200476

$\mathbf{L}$ 'isolement volontaire des personnes présentant des symptômes bénins de maladie à coronavirus 2019 (COVID-19), le placement en quarantaine des personnes exposées et l'éloignement social du reste de la population constituent la réalité canadienne d'aujourd'hui. De nouvelles études ont modélisé les effets probables de l'isolement volontaire ${ }^{1}$ et des diverses méthodes d'éloignement social ${ }^{2}$ sur les besoins de soins intensifs au Canada, et les résultats sont peu réjouissants.

Shoukat et ses collaborateurs ont découvert que sans mesures d'éloignement social, même le respect total des directives d'isolement volontaire par les personnes ayant contracté le coronavirus du syndrome respiratoire aigu sévère 2 (SRAS-CoV-2) ne préviendra pas la surcharge des services de soins intensifs dans les provinces canadiennes ${ }^{1}$. Tuite et ses collaborateurs, qui ont modélisé des données ontariennes et se sont plutôt penchés sur les effets de l'éloignement social, ont montré que seule l'adoption à long terme de mesures d'éloignement social strictes aplatira la courbe épidémiologique². Ils ont proposé 2 modèles sur 2 ans : un dans lequel les mesures sont fixes, restant les mêmes au fil du temps, et un dans lequel elles sont dynamiques et cycliques, étant relâchées ou renforcées selon le taux d'occupation des lits de soins intensifs.

L'éloignement social dynamique consiste à réagir à la courbe épidémiologique en permettant au nombre de cas de COVID-19 de fluctuer, à condition que le fardeau pour les services de soins intensifs demeure gérable. Tuite et ses collaborateurs considèrent que cette approche permettrait d'éviter la surcharge des systèmes de santé, mais atténuerait également les effets psychologiques et les effets néfastes pour la santé de l'éloignement social prolongé, et pourrait même possiblement atténuer certaines répercussions économiques.

L'éloignement social sur une longue durée sera difficile, mais les meilleures estimations en date d'aujourd'hui montrent clairement que si nous ne le faisons pas, les systèmes de santé canadiens s'effondreront, comme ceux de l'Italie et de New York. Et si nous ne le faisons pas correctement, il y aura trop de décès évitables, de deuils nationaux et de travailleurs de la santé devant prendre des décisions déchirantes sur le plan moral, c'est-à-dire choisir qui aura accès aux ressources vitales. Les résultats des études de modélisation mettent en évidence un argument souvent soulevé au cours des dernières semaines : les travailleurs de la santé ne sont pas la première ligne de défense, mais plutôt la dernière.

Bien que certaines personnes se soient rapidement adaptées à la nouvelle réalité - elles respectent par exemple les consignes d'éloignement physique à l'épicerie ou travaillent à la maison -, l'éloignement social est déjà insoutenable pour les personnes qui n'ont pas de domicile où se réfugier, celles qui pourraient subir de la violence à la maison et celles qui ont perdu leur emploi. Les communautés devront trouver des moyens d'aider les personnes vulnérables à s'isoler volontairement ou à respecter les mesures d'éloignement social.

Les politiciens et les responsables de la santé publique canadiens, qui dans certains cas s'opposaient farouchement les uns aux autres, semblent bien collaborer pour diffuser des messages cohérents. Or, compte tenu des nouvelles données, les dirigeants devront annoncer clairement à la population canadienne que l'éloignement social durera probablement beaucoup plus longtemps que prévu. Si les membres des communautés sont notre première ligne de défense, il faudra absolument faire preuve de transparence dans la présentation des données et reconnaître les incertitudes pour préserver leur confiance.

Tous les modèles sont limités par les hypothèses et les données sur lesquelles ils reposent. Ce fait est d'ailleurs bien illustré par les 2 études présentées, dans lesquelles les auteurs ont avancé des hypothèses très différentes et se sont servi d'ensembles de données publiées à divers moments. Il se pourrait que la capacité de dépistage accru du SRAS-CoV-2 au Canada change les données utilisées pour la modélisation et donne lieu à de nouvelles projections, ou que le taux d'atteinte de la maladie chez les professionnels de la santé modifie les hypothèses sur la capacité des services de soins intensifs. Cependant, la planification reposant sur les résultats de modèles limités est préférable à la planification ne reposant sur rien du tout. 
Comme il est peu probable qu'un vaccin soit largement accessible avant quelques années ${ }^{3}$ et qu'aucun traitement antiviral efficace n'est actuellement disponible pour contrecarrer la maladie, la COVID-19 représentera longtemps une menace pour les systèmes de santé canadiens. Ces 2 modèles bien différents montrent que l'adoption de courte durée et le respect partiel de mesures de santé publique ne feront que retarder la surcharge des services de soins intensifs. Ainsi, il est temps que les dirigeants parlent franchement au public de l'adoption de mesures de plus longue durée et lui expliquent clairement ce qui l'attend. Il est aussi temps que les gouvernements mettent en place diverses initiatives, comme loger les personnes sans-abri à l'hôtel ou combler le manque à gagner des travailleurs, pour que la population canadienne puisse vivre un éloignement social sûr et le plus facile possible. Enfin, il est temps que les personnes qui appliquent les mesures d'éloignement social comprennent le rôle important qu'elles jouent en première ligne. Les travailleurs de la santé et les futurs patients ont besoin que chacun d'entre nous contribue à freiner la propagation du SRAS-CoV-2.

\section{Références}

1. Shoukat A, Wells CR, Langley JM, et al. Projecting demand for critical care beds during COVID-19 outbreaks in Canada. CMAJ le 8 avril 2020 [Cyberpublication avant impression]. doi: 10.1503/200457.

2. Tuite AR, Fisman DN, Greer AL, et al. Mathematical modelling of COVID-19 transmission and mitigation strategies in the population of Ontario, Canada. CMAJ le 8 avril 2020 [Cyberpublication avant impression]. doi: 10.1503/200476.

3. Lurie N, Saville M, Hatchett R, et al. Developing COVID-19 vaccines at pandemic speed. N Engl J Med le 30 mars 2020 [Cyberpublication avant impression]. doi: 10.1056/NEJMp2005630.

Intérêts concurrents : Voir www.cmaj.ca/site/misc/cmaj_staff.xhtml

Affiliations : Directrice de la rédaction, CMAJ (Patrick); rédacteur adjoint, CMAJ (Stanbrook); Département de médecine (Stanbrook), Université de Toronto, Toronto, Ont.; rédacteur en chef, CMAJ (Laupacis)

Correspondance : Rédaction du CMAJ, cmaj@cmajgroup.ca 